\title{
Immunohistochemical evidence for involvement of inflammatory cytokines in anti-arrhythmic effects of rosuvastatin in male rats
}

\author{
Sima Amini ${ }^{1}$, Vahid Nikoui ${ }^{2}$, Farahnaz Jazaeri ${ }^{1}$, Muhammad Imran Khan ${ }^{3}$, Alireza Partoazar ${ }^{4}$, Azam \\ Bakhtiarian $^{1,4 *}$ iD \\ 1. Department of Pharmacology, School of Medicine, Tehran University of Medical Sciences, Tehran, Iran \\ 2. Razi Drug Research Center, Iran University of Medical Sciences, Tehran, Iran \\ 3. Department of Pharmacy, Kohat University of Science and Technology, 26000 Kohat, Pakistan \\ 4. Experimental Medicine Research Center, Tehran University of Medical Sciences, Tehran, Iran
}

\begin{abstract}
Introduction: Considering the cardioprotective and anti-inflammatory properties of statins, the aim of the present experiment was to investigate the possible involvement of inflammatory cytokines in anti-arrhythmic effects of rosuvastatin in both in vitro and in vivo experiments in rats.
\end{abstract}

Methods: Three weeks after oral administration of either of rosuvastatin or vehicle, the atria were removed and after incubation with ouabain, time of onset of arrhythmia and asystole were recorded. We also used immunohistochemistry technique to identify the differentially expressed proteins interleukin (IL)-1 $\beta$, IL-6 and tumor necrosis factor (TNF)- $\alpha$ in atria.

Results: Rosuvastatin significantly postponed the onset of arrhythmia compared to vehicle-treated group. Injection of ouabain increased the atrial expression of IL-1 $\beta$, IL6 and TNF- $\alpha$ proteins, while pretreatment of rats with rosuvastatin could significantly attenuate them.

Conclusion: Our data suggest that rosuvastatin exerts anti-arrhythmic properties at least in part through modulation of inflammatory cytokines.

http://dx.doi.org/10.32598/ppj.24.2.60

\section{Keywords:}

Rosuvastatin;

Cardiovascular;

Arrhythmia;

Inflammatory cytokines

\section{* Corresponding author:}

\section{A. Bakhtiarian \\ Email:}

bakhtiar@tums.ac.ir

Tel: +98 (21) 64053215

Received 4 November 2019; Received in revised form 27 February 2020; Accepted 10 March 2020

\section{Introduction}

Since most anti-arrhythmic drugs possess serious adverse effects, finding safer anti-arrhythmic agents with fewer side effects is very important. Statins are the main class of drugs, which used for management of hypercholesterolemia (Gagné et al., 2002). Studies have revealed that statins possess various additional beneficial cardioprotective properties against stroke, angina and thrombosis (Sever et al., 2003, Law et al., 2003, Wilson et al., 1998, Furie, 2012). Pleiotropic effects of statins on arrhythmia have also been reported (Turagam et al., 2015) based on the mechanisms including the improvement of heart rate variability (Pehlivanidis et al., 2001), the reduction in corrected QT duration and QT variability (Vrtovec et 
al., 2005) and the inhibition of ventricular late potentials (Kayikcioglu et al., 2003). Statins also diminish the risk of ventricular tachyarrhythmia and sudden cardiac death both in coronary artery disease and non-ischemic cardiomyopathy (Buber et al., 2012, Dickinson et al., 2007, Bourne et al., 2010). In addition, statin therapy possesses a beneficial effect on aortic arterial stiffness (Upala et al., 2017) and could diminish the overall mortality, significantly (Nunes, 2017). Frequent experiments report that statins at high doses also have anti-inflammatory effects and improve endothelial function, which can stabilize and even regress plaques (Jain and Ridker, 2005, Weitz-Schmidt, 2002, Diomede et al., 2001, Libby and Aikawa, 2003, Lima et al., 2004). On the other hand, evidence shows that inflammatory cytokines have a key role in occurrence of various cardiac arrhythmias (Marcus et al., 2010, Engelmann and Svendsen, 2005, Duncan et al., 2010).

Ouabain is a cardiac glycoside, which has positive inotropic effects and can improve the heart failure treatment through inhibition of the $\mathrm{Na}^{+} / \mathrm{K}^{+}$-ATPase and $\mathrm{Na}^{+} / \mathrm{Ca}^{2+}$ exchanger and subsequent increase of intracellular calcium and myocardial contraction ( $\mathrm{Yu}$ and Choi, 1997). Nevertheless, higher doses of ouabain represents an animal model for induction of cardiac arrhythmias. On the other hand, it has been reported that ouabain induces the production of proinflammatory cytokines (Matsumori et al., 2000).

Since a majority of hyperlipidemic patients suffer from comorbid cardiovascular disorders including cardiac arrhythmias and considering the cardioprotective properties of statins, we previously reported that atorvastatin exerts marked anti-arrhythmic properties through diminution of inflammatory cytokines levels in atria (Najjari et al., 2018). Subsequently, in the present experiment, we aimed to detect the atrial immunostaining reactions of inflammatory cytokines in possible anti-arrhythmic properties of another drug of statin family, rosuvastatin in ouabain-induced arrhythmia in isolated rat atria.

\section{Materials and methods}

\section{Animals}

Male Wistar albino rats weighting 200-230g were used. Rats were kept at the temperature of $22 \pm 2^{\circ} \mathrm{C}$, humidity $80 \%$, 12-h light/dark cycle (light on at $7 \mathrm{am}$ ) and had ad libitum access to food and water. All experiments were conducted in Tehran University of
Medical Sciences in accordance with the recommendations of the ethics committee on animal experimentation of the medical school (number IR. TUMS.MEDICINE.REC.1396.4132), which conforms to the provisions of the Declaration of Helsinki.

\section{Chemicals}

Salts for preparing physiological salt solution were purchased from Merck (Germany). Rosuvastatin, ouabain and 4',6-diamidino-2-phenylindole (DAPI) were prepared from Sigma-Aldrich (USA) and immunohistochemical antibodies against interleukin (IL)-1 $\beta$, IL-6 and tumor necrosis factor (TNF)- $\alpha$ were purchased from Abcam (USA).

\section{Experimental groups In vitro experiment}

For isolated atria recording, 12 rats divided into two equal groups received either of $10 \mathrm{mg} / \mathrm{kg}$ rosuvastatin dissolved in $1 \%$ carboxymethyl cellulose (CMC) in normal saline as vehicle or only $1 \%$ CMC in normal saline (Wallace et al., 2000), orally once daily for 3 weeks. Rosuvastatin or CMC were administered by oral gavage in volume of $1 \mathrm{ml} / \mathrm{kg}$, once daily. In this procedure, a stainless steel bulb tipped gavage needle was attached to a syringe and used to deliver the compound into the stomach.

\section{In vivo experiment}

For immunohistochemical studies, 12 new rats different from the aforementioned rats were divided into three equal groups. The first and second groups received vehicle ( $1 \% \mathrm{CMC})$, while the third group was pretreated with rosuvastatin $(10 \mathrm{mg} / \mathrm{kg}$ in $1 \% \mathrm{CMC}$ ). Rosuvastatin or CMC were administered by oral gavage in volume of $1 \mathrm{ml} / \mathrm{kg}$, once daily for 3 weeks. From the days 19-21, the first group was injected by normal saline, whereas the second and third groups received injection of ouabain $(0.56 \mathrm{mg} / \mathrm{kg}$ ) (RodriguesMascarenhas et al., 2006). All injections were in volume of $1 \mathrm{ml} / \mathrm{kg}$ once daily for three consecutive days, intraperitoneally.

\section{Recording of isolated atria}

After anesthesia with ketamine $(80 \mathrm{mg} / \mathrm{kg}$, Alfasan, Netherlands) and diazepam $(2 \mathrm{mg} / \mathrm{kg}$, Caspian Tamin, Iran), the atria was rapidly removed and immersed in a tissue bath containing $20 \mathrm{ml}$ of carbogenated $(95 \%$ $\mathrm{O}_{2}$ and $5 \% \mathrm{CO}_{2}$ ) physiological slat solution at $37^{\circ} \mathrm{C}$ 
and pH 7.4 (Ghebleh Zadeh et al., 2018). The composition of the solution was as follows $(\mathrm{mM})$ : $\mathrm{NaCl} 112.0, \mathrm{KCl} 5, \mathrm{CaCl}_{2} 1.8, \mathrm{MgCl}_{2} 1.0, \mathrm{NaH}_{2} \mathrm{PO}_{4}$ 0.5, $\mathrm{KH}_{2} \mathrm{PO}_{4}$ 0.5, $\mathrm{NaHCO}_{3}$ 25.0, glucose 10.0 and EDTA 0.004 (Merck, Germany). After 30min equilibration under a preload tension of $1000 \mathrm{mg}$, we recorded the onset time of arrhythmia (bigeminy) and asystole following incubation with ouabain $(40 \mu \mathrm{M})$ (Ghebleh Zadeh et al., 2018) as well as atrial beating rate and contractile force using isometric force transducer of PowerLab system (ADInstrument, Australia) and LabChart software.

\section{Immunohistochemistry}

Immunohistochemical study was carried out based on previous report (Cai and Wang, 2017). Briefly, paraffin sections of atrial tissue samples (5 micron thickness) were prepared. After dewaxed, tissue sections were washed in phosphate buffered saline (PBS, PH 7.4) for four times, followed by antigen retrieval by adding $\mathrm{HCl} 2 \mathrm{~N}$ (normal) for $30 \mathrm{~min}$. A primary antibody against IL-1 $1 \beta$, IL- 6 or TNF- $\alpha$ (1:100 dilution) was added. After PBS wash, secondary antibody (Ab6785, Abcam, USA. 1:150 dilution) was added. Then, the fluorescent stain DAPI was added and examined under the fluorescent microscope (Olympus, Japan, 400X). The expression level was observed and analyzed in Image-pro plus system.
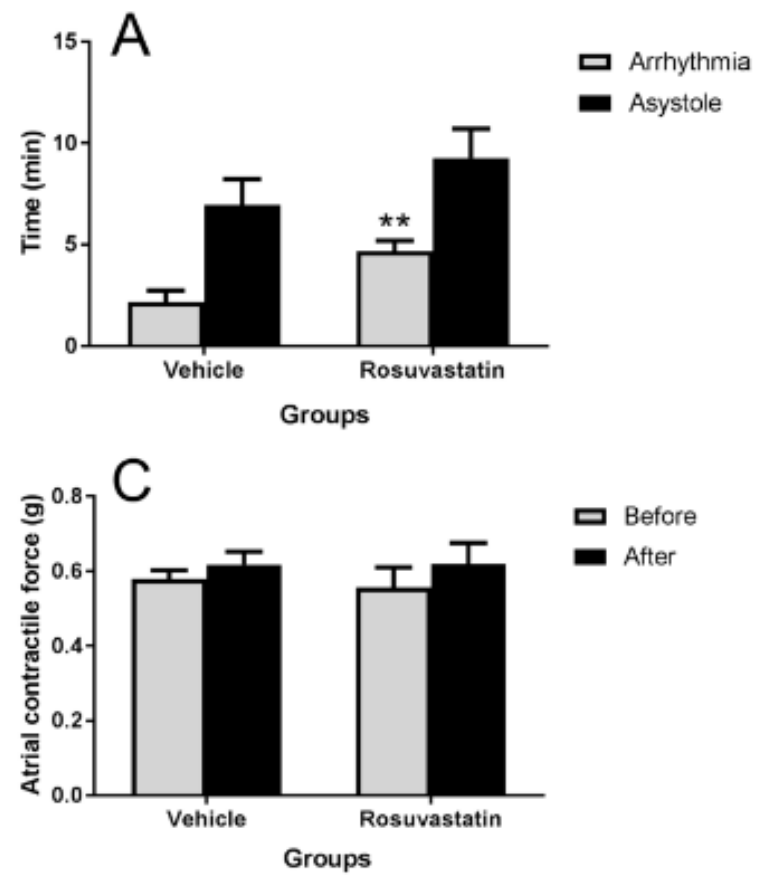

\section{Statistical analysis}

GraphPad Prism 5 software (San Diego, CA) was used for statistical analyses. Results are shown as mean \pm SEM. We used unpaired Student's t-test to compare the onset time of arrhythmia and asystole and atrial beating rate and contractile force between treatment and control groups. We also used paired Student's t-test to determine the effects of ouabain on atrial beating rate and contractile force within groups. One-way analysis of variance (ANOVA) followed by Tukey's post-hoc test was carried out to compare the atrial expression of inflammatory cytokines between groups. $P$-values less than 0.05 were considered statistically significant.

\section{Results}

\section{Recording of isolated atria}

Pretreatment of rats with rosuvastatin postponed the time of onset of arrhythmia (4.67min) compared to control vehicle-treated group (2.18min, $P \leq 0.01)$. Although the time of onset of asystole in rosuvastatintreated group $(9.27 \mathrm{~min})$ seems longer than control (6.95min), no statistically significant difference was detected between treatment and control groups $(P>0.05$, Fig. $1 A)$. The difference in atrial beating rate between treatment (303.62 beat $/ \mathrm{min}$ before, and significant $(P>0.05$, Fig. 1B). Similarly, there was no statistically significant difference between atrial

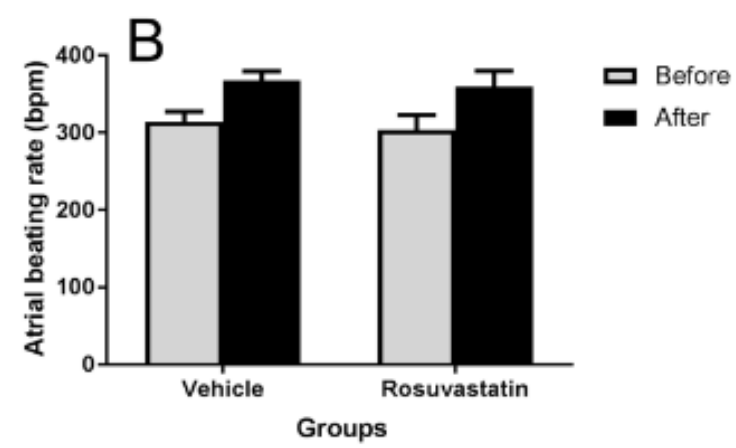

Fig.1. Time of onset of arrhythmia and asystole (A), atrial beating rate (B) and atrial contractile force (C) after ouabain incubation in vehicle and rosuvastatin-treated groups. Data are shown as mean \pm SEM. Six rats were used in each group. ${ }^{* *} P \leq 0.01$ compared to arrhythmia of vehicle-treated group. 

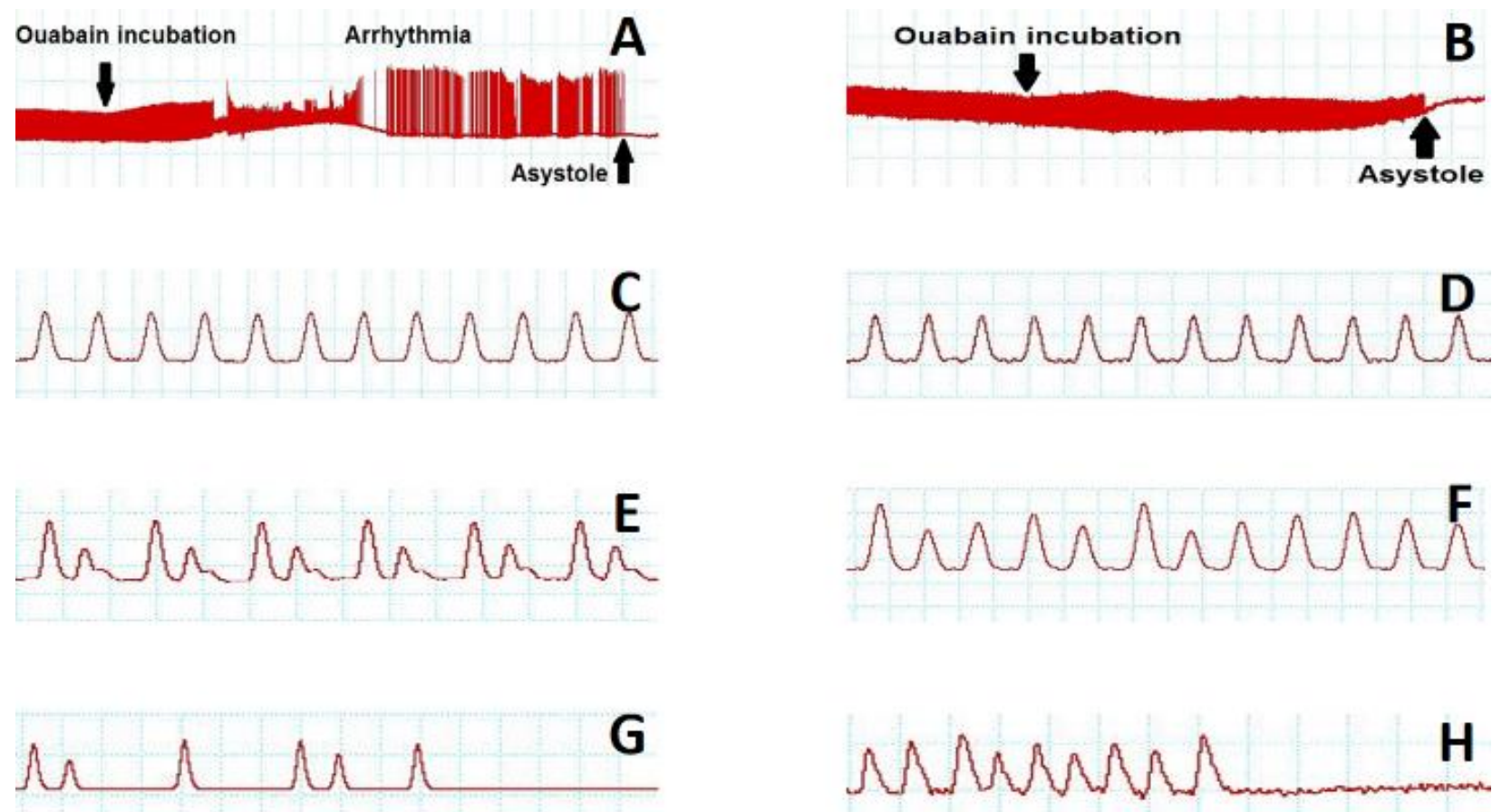

Fig.2. Chronotropic and inotropic pattern in vehicle and rosuvastatin-treated groups. In control group, ouabain-induced arrhythmia was strong (A), while the intensity of arrhythmia in rosuvastatin-treated group was diminished (B). The atrial beatings and contractile force prior incubation of ouabain were similar in both groups (C, D). Bigeminy arrhythmias (twin spikes with strong force), which is the typical manifestation of ouabain-induced arrhythmia were seen in control group (E), while in rosuvastatin-treated group, some small irregularities were recorded (F). The onset time of asystole in rosuvastatintreated group $(\mathrm{H})$ was later than control group $(\mathrm{G})$.
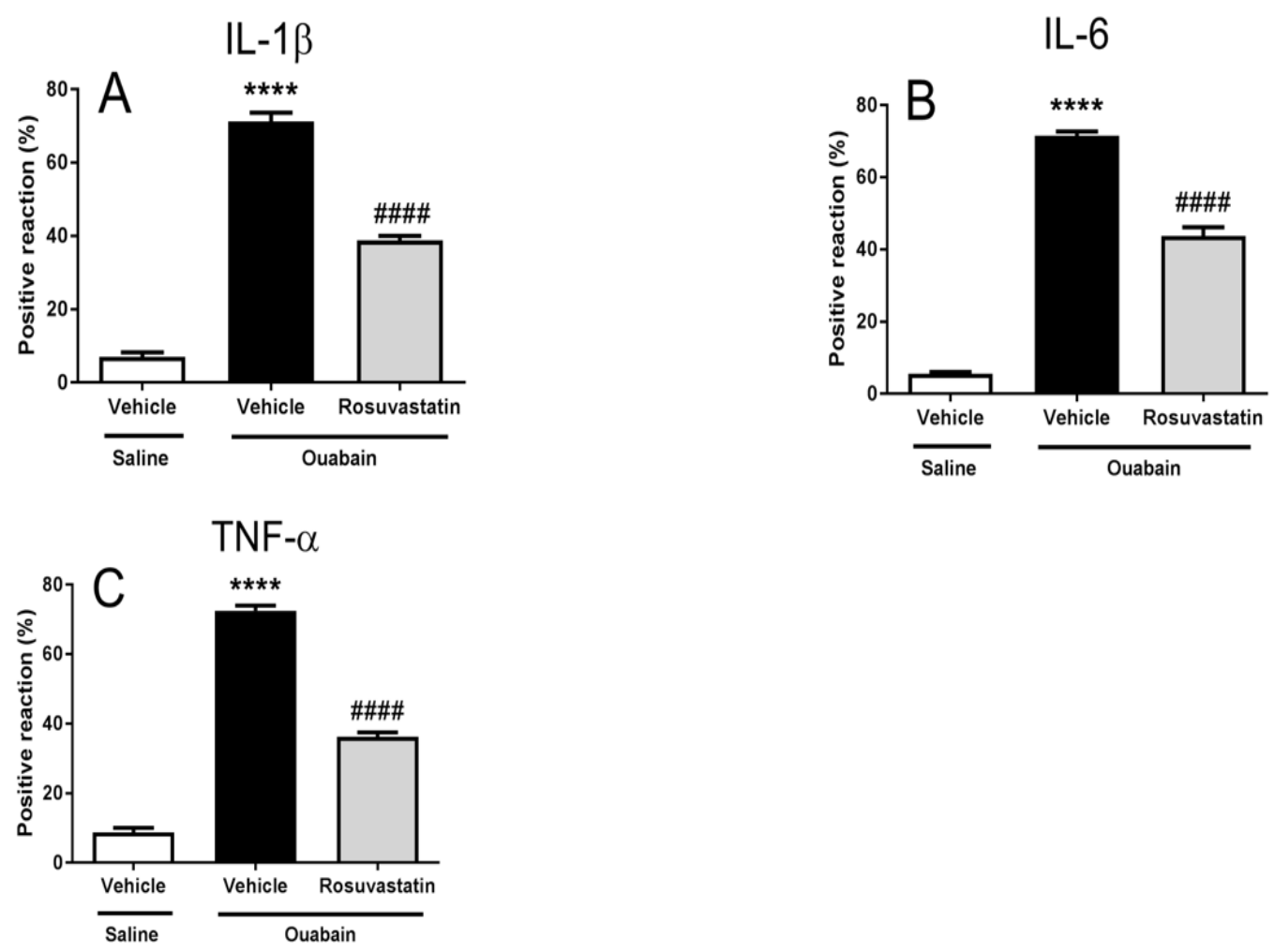

Fig.3. Atrial levels of IL-1 $\beta(A), I L-6(B)$ and TNF- $\alpha(C)$ in saline and ouabain-injected vehicle and rosuvastatin-treated groups. Data are shown as mean \pm SEM. Four rats were used in each group. ${ }^{\star \star \star *} P \leq 0.0001$ compared to saline-injected vehicle-treated group; ${ }^{\# \# \# ~} P \leq 0.0001$ compared to ouabain-injected vehicle-treated group. 


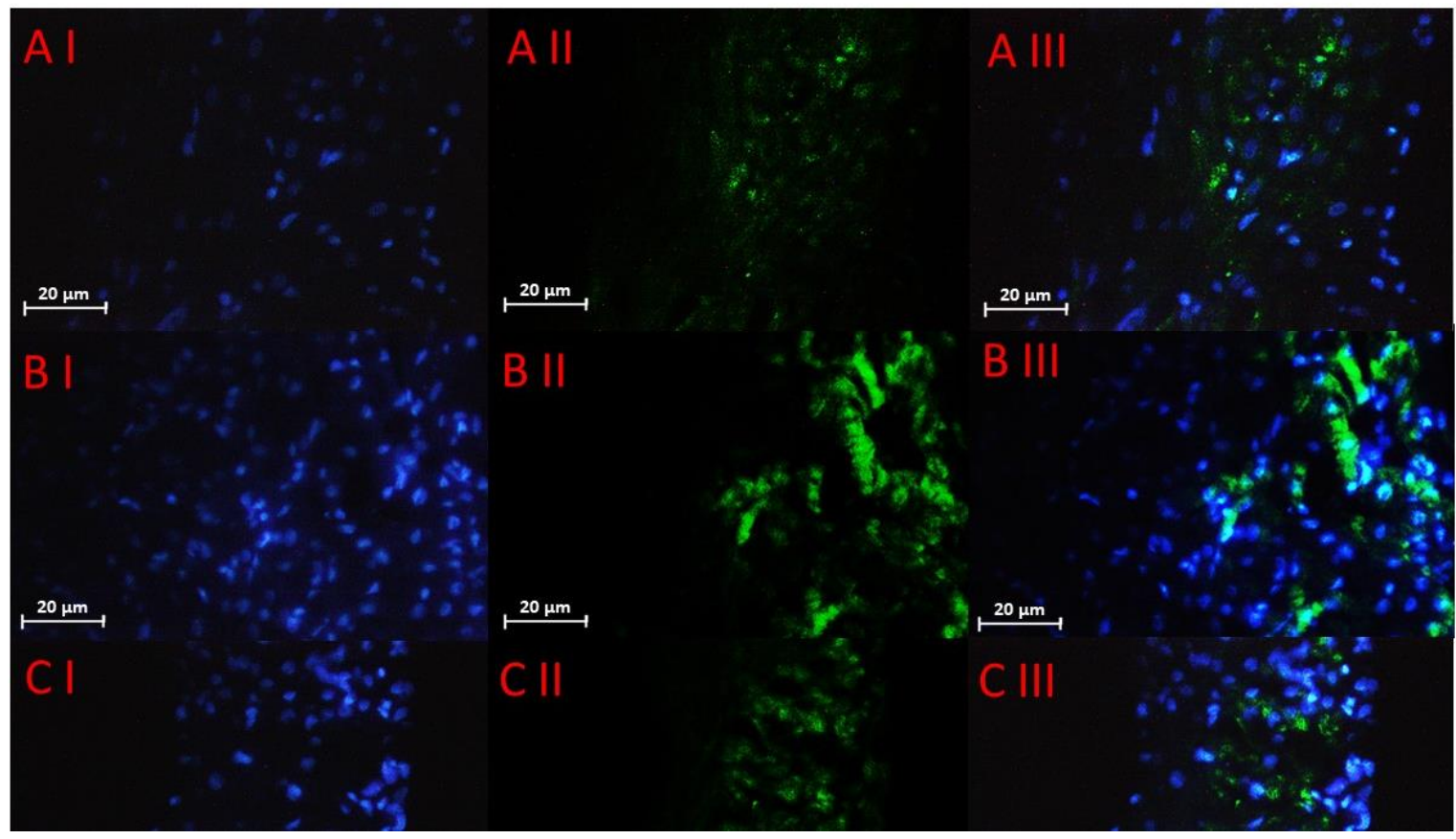

Fig.4. Representative immunostaining of $\mathrm{IL}-1 \beta$ in atrial tissue arrays of saline and ouabain-injected vehicle and rosuvastatin-treated groups. A: vehicle+ saline, positive reaction $10 \%$. B: vehicle+ ouabain, positive reaction $75 \%$. C: rosuvastatin+ ouabain, positive reaction $40 \%$. I: nuclei stained by DAPI; II: primary antibody to IL-1 $1 \beta$; III: merge. Magnification 400X.

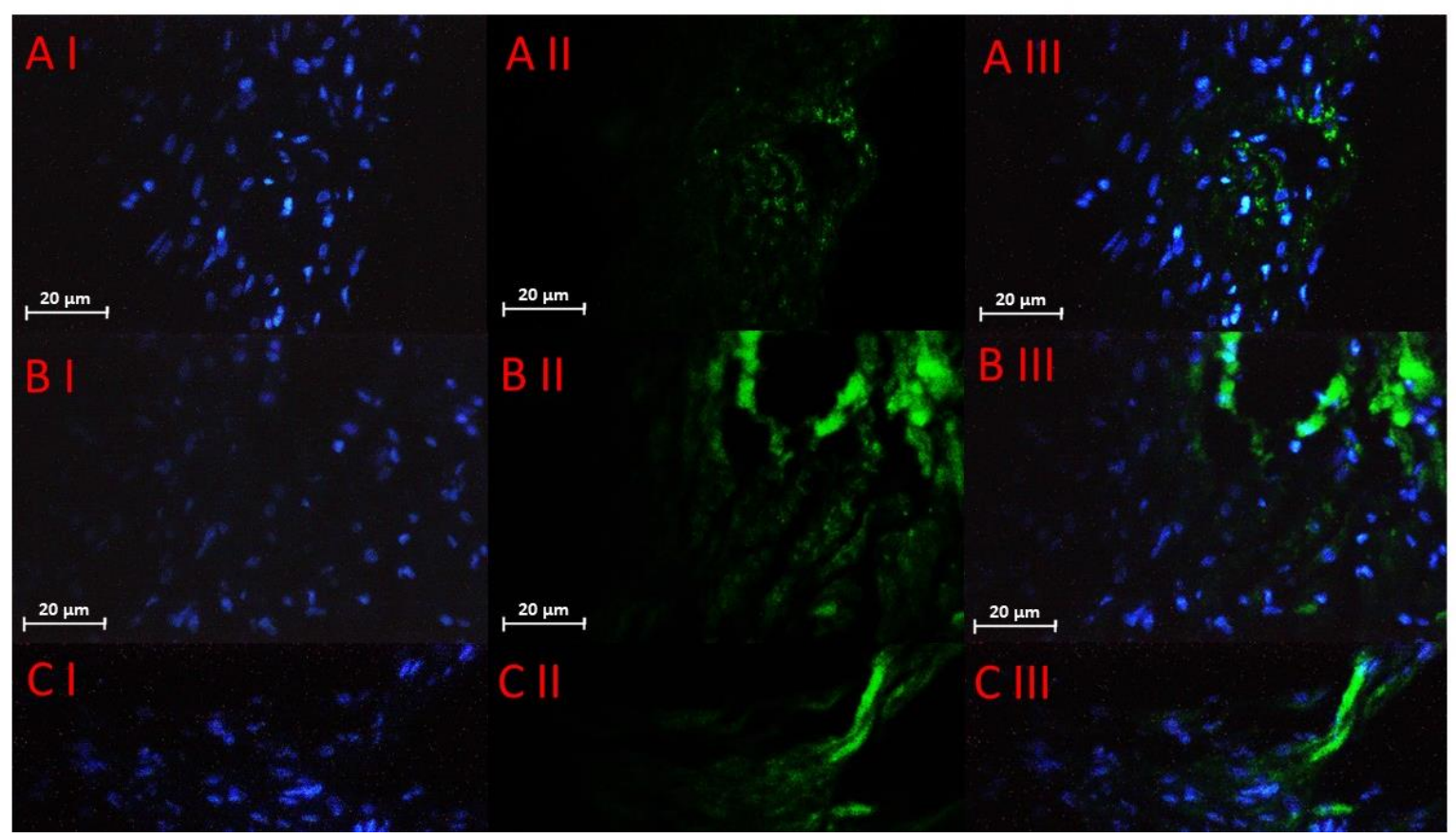

Fig.5. Representative immunostaining of IL-6 in atrial tissue arrays of saline and ouabain-injected vehicle and rosuvastatintreated groups. A: vehicle+ saline, positive reaction 7\%. B: vehicle+ ouabain, positive reaction $75 \%$. C: rosuvastatin+ ouabain, positive reaction 40\%. I: nuclei stained by DAPI; II: primary antibody to IL-6; III: merge. Magnification 400X.

contractile force of control $(0.57 \mathrm{~g}$ before, and $0.61 \mathrm{~g}$ after ouabain incubation) and treatment $(0.55 \mathrm{~g}$ before, and $0.61 \mathrm{~g}$ after ouabain incubation) groups ( $P>0.05$, Fig.1C). Figure 2 compares the chronotropic and inotropic recording pattern in vehicle and rosuvastatin-treated groups. In control group, incubation of ouabain showed dramatic arrhythmias (Fig. 2A), while pretreatment of rats with rosuvastatin $359.63 \mathrm{beat} / \mathrm{min}$ after ouabain incubation) and control (314.55 beat/min before, and 367.82 beat/min after 


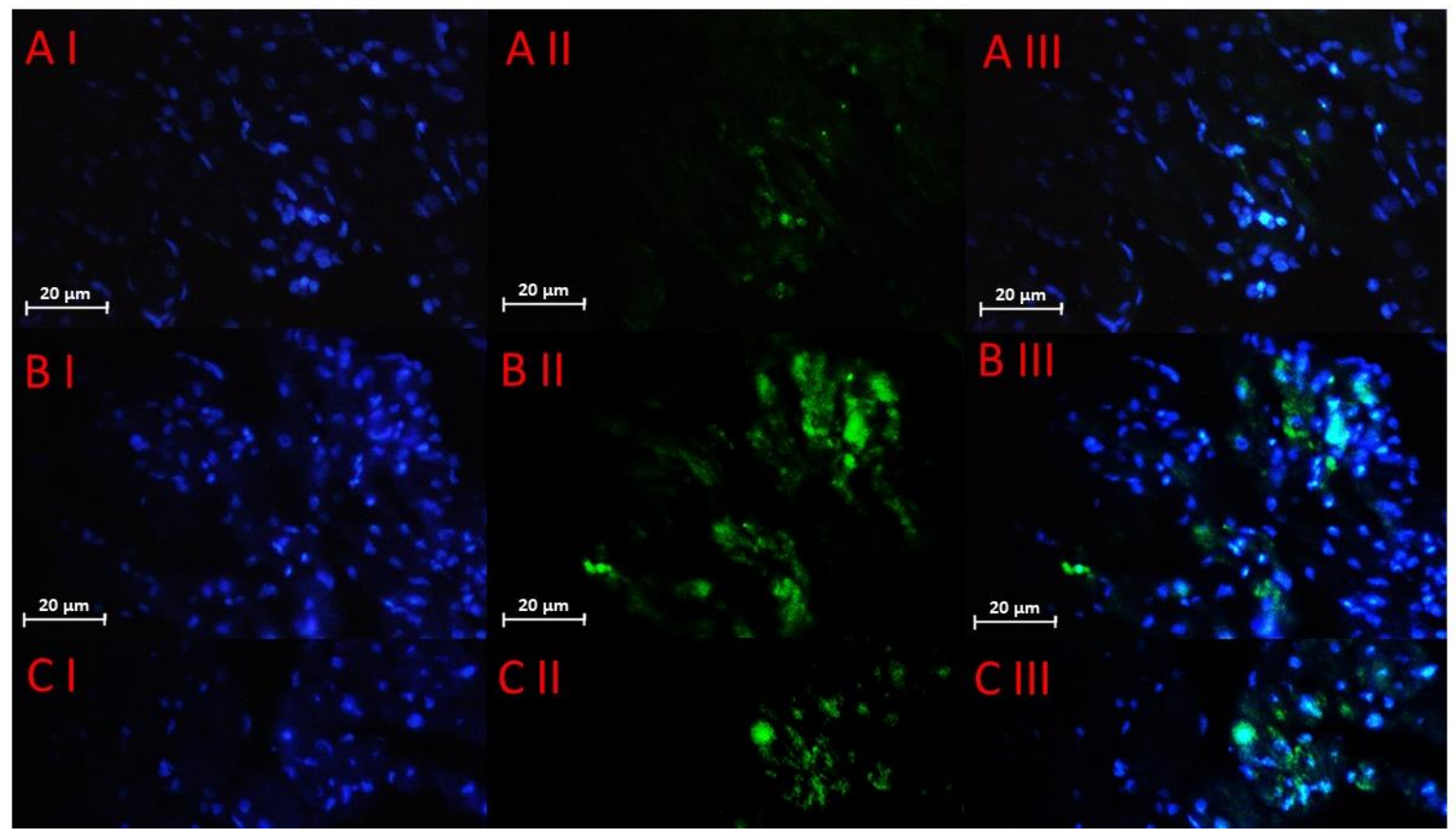

Fig.6. Representative immunostaining of TNF- $\alpha$ in atrial tissue arrays of saline and ouabain-injected vehicle and rosuvastatin-treated groups. A: vehicle+ saline, positive reaction $10 \%$. B: vehicle+ ouabain, positive reaction $75 \%$. C: rosuvastatin+ ouabain, positive reaction $40 \%$. I: nuclei stained by DAPI; II: primary antibody to TNF-a; III: merge. Magnification 400X.

ouabain incubation) groups was not statistically alleviated the intensity of arrhythmias (Fig. 2B). The shape of arrhythmia in control group was typical bigeminy (twin spikes with strong force, repeatedly), which is the common manifestation of ouabaininduced arrhythmia (Fig. 2E). This arrhythmia lasted for several minutes and returned to the regular atrial rhythm in some samples. In rosuvastatin-treated group, irregularities between normal spikes were seen (Fig. 2F) and they occurred much later than control group.

\section{Immunohistochemistry}

Injection of ouabain significantly boosted the atrial expression levels of all studied inflammatory cytokines $(P \leq 0.0001)$. On the other hand, pretreatment of rats with rosuvastatin could attenuate them, significantly ( $P \leq 0.0001$, Fig. 3 ). Figure 4 shows the representative immunostaining of $\mathrm{IL}-1 \beta$ in atrial tissue arrays of saline and ouabain-injected vehicle and rosuvastatin-treated groups. The positive reaction in saline-injected vehicle-treated group was $10 \%$, while injection of ouabain elevated it to $75 \%$ and pretreatment of rats with rosuvastatin could significantly diminish it to $40 \%$. Illustrating in Figure 5 , the positive reaction for IL- 6 in saline-injected vehicle- treated group was only $7 \%$, while injection of ouabain boosted it to $75 \%$ and pretreatment of rats with rosuvastatin could significantly attenuate it to $40 \%$. Similarly, the positive reaction for TNF- $\alpha$ in salineinjected vehicle-treated group was $10 \%$, while injection of ouabain increased it to $75 \%$ and pretreatment of rats with rosuvastatin could significantly decrease it to $40 \%$ (Fig. 6).

\section{Discussion}

In the present experiment, we studied the involvement of inflammatory cytokines in antiarrhythmic effects of rosuvastatin in both in vitro and in vivo examinations in rats. Results showed that administration of rosuvastatin delayed the onset of ouabain-induced arrhythmia. Furthermore, rosuvastatin attenuated the ouabain-induced overexpression of inflammatory cytokines.

Evidence show that ouabain boosts the production of pro-inflammatory cytokines (Chung et al., 2001, Vila et al., 2000, Vasan et al., 2003). For example, Matsumori et al. (2000) have reported that incubation

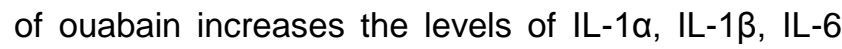
and TNF- $\alpha$ in cultured human peripheral blood mononuclear cells. We also previously showed that ouabain could trigger the production of inflammatory 
cytokines in rat atria (Tokazzabani Belasi et al., 2018, Najjari et al., 2018, Moradi et al., 2016). It is also reported that atrial fibrillation increases the production of IL-6 in patients (Ishida, 2006). Our data are in agreement with the aforementioned experiments. Besides, we also showed that rosuvastatin attenuates the over-production of inflammatory cytokines following ouabain injection, which corroborates our previous experiment on atorvastatin (Najjari et al., 2018).

The metabolic syndrome can be associated with increased risk of atrial fibrillation and other cardiac arrhythmias (Watanabe et al., 2008). Statins exert their anti-arrhythmic properties through reduction in corrected QT duration, inhibition of ventricular late potentials and improvement of heart rate variability (Pehlivanidis et al., 2001, Vrtovec et al., 2005, Kayikcioglu et al., 2003). Perioperative statin therapy is a therapeutic strategy for prevention of postoperative atrial fibrillation via inhibiting the inflammatory pathways (Sanchez-Quinones et al., 2008). Statins possess beneficial effects in acute myocardial infarction (Sicard et al., 2007, Baigent et al., 2008, Lenderink et al., 2006). The American heart association, American college of cardiology and European society of cardiology all suggest that statins should be prescribed following acute myocardial infarction (O'gara et al., 2013).

A recent clinical trial study in 2018 compared the effects of hydrophilic rosuvastatin and lipophilic atorvastatin on nonsustained ventricular tachycardia. They suggested that the effects of rosuvastatin on nonsustained ventricular tachycardia might be better than that of atorvastatin (Hu et al., 2018). Evidence show that adverse events of statins are dosedependent (Jones et al., 2003). Given that a lower dose of rosuvastatin has a similar effect to higher doses of other statins, it is beneficial to use it (Guo et al., 2017). A high-dose of rosuvastatin can postpone ventricular remodeling, effectively suppress malignant remodeling of the heart, improve left ventricular systolic function, diminish the prevalence of adverse events and significantly improve the long-term prognosis (Guo et al., 2017).

Numerous experiments have reported the antiinflammatory effects of statin (Blanco-Colio et al., 2003, S Antonopoulos et al., 2012, Mehrzadi et al., 2015). Inflammatory cytokines play a dramatic role in various pathological disorders. For example, London et al. (2003) have reported that TNF- $\alpha$ triggers the incidence of various atrial and ventricular arrhythmias. There are also other experiments indicating the involvement of TNF- $\alpha$ in pathophysiology of ventricular arrhythmia (Kowalewski et al., 2002, Duncan et al., 2010). Similarly, in the present experiment, our results are in agreement with the aforementioned reports indicating the anti-inflammatory properties of rosuvastatin. Moreover, various clinical trials report a marked correlation between atrial fibrillation and high levels of IL-6 (Ishida, 2006, Psychari et al., 2005, Wu et al., 2013). The ouabain-induced over-expression of IL-6 in our study also confirms the previous experiment.

Clinical practice guidelines suggest that cardiovascular benefits of statins generally outweigh non-cardiovascular adverse effects in patients suffering from cardiovascular disorders (Desai et al., 2014). The potentials of diverse therapeutic indications of statins could introduce the novel applications of this class of drugs in the future.

\section{Conclusion}

Our data suggest that rosuvastatin possesses antiarrhythmic properties and diminution of atrial inflammatory cytokines expression might at least in part explain this response. Possible other mechanisms for anti-arrhythmic effects of rosuvastatin need for further experiments.

\section{Acknowledgments}

This research was resulted from a M.D. thesis and has been supported by Tehran University of Medical Sciences (TUMS), grant number 96-03-30-36328.

\section{Conflict of interest}

None of the authors of this paper has a financial or personal relationship with other people or organizations that could inappropriately influence or bias the content of the paper.

\section{References}

Baigent C, Keech A, Kearney PM, Blackwell L, Buck G, Pollicino $C$, et al. Efficacy and safety of cholesterollowering treatment: prospective meta-analysis of data from 90,056 participants in 14 randomised trials of statins. Lancet 2005; 366: 1267-78. https://doi.org/ 10.1016/S0140-6736(05)67394-1

Blanco-Colio LM, Tuñón J, Martín-Ventura JL, Egido J. Anti-inflammatory and immunomodulatory effects of 
statins. Kidney Int 2003; 63: 12-23. https://doi.org/ 10.1046/j.1523-1755.2003.00744.x

Bourne PA, Francis CG, Kerr-Campbell MD. 2010. Patient care: Is interpersonal trust missing?. N Am J Med Sci 2010; 2: 126-33.

Buber J, Goldenberg I, Moss AJ, Wang PJ, McNitt S, Hall WJ, et al. Reduction in life-threatening ventricular tachyarrhythmias in statin-treated patients with nonischemic cardiomyopathy enrolled in the MADITCRT (Multicenter Automatic Defibrillator Implantation Trial with Cardiac Resynchronization Therapy). J Am Coll Cardiol 2012; 60: 749-55. https://doi.org/10.1016/ j.jacc.2012.03.041

Cai YL, Wang ZW. The expression and significance of IL-6, IFN- $\gamma, S M 22 \alpha$, and MMP-2 in rat model of aortic dissection. Eur Rev Med Pharmacol Sci 2017; 21: 560568.

Chung MK, Martin DO, Sprecher D, Wazni O, Kanderian A, Carnes CA, et al. C-reactive protein elevation in patients with atrial arrhythmias: inflammatory mechanisms and persistence of atrial fibrillation. Circulation 2001; 104: 2886-91. https://doi.org/10.1161/ hc4901.101760

Desai CS, Martin SS, Blumenthal RS. Non-cardiovascular effects associated with statins. BMJ 2014; 349: 1-10. https://doi.org/10.1136/bmj.g3743

Dickinson MG, Ip JH, Olshansky B, Hellkamp AS, Anderson $\mathrm{J}$, Poole JE, et al. Statin use was associated with reduced mortality in both ischemic and nonischemic cardiomyopathy and in patients with implantable defibrillators: mortality data and mechanistic insights from the Sudden Cardiac Death in Heart Failure Trial (SCD-HeFT). Am Heart J 2007; 153: 573-8. https://doi. org/10.1016/j.ahj.2007.02.002

Diomede L, Albani D, Sottocorno M, Donati MB, Bianchi M, Fruscella $P$, et al. In vivo anti-inflammatory effect of statins is mediated by nonsterol mevalonate products. Arterioscler Thromb Vasc Biol 2001; 21: 1327-32. https://doi.org/10.1161/hq0801.094222

Duncan DJ, Yang Z, Hopkins PM, Steele DS, Harrison SM. TNF-alpha and IL-1beta increase $\mathrm{Ca} 2+$ leak from the sarcoplasmic reticulum and susceptibility to arrhythmia in rat ventricular myocytes. Cell Calcium 2010; 47: 37886. https://doi.org/10.1016/j.ceca.2010.02.002

Engelmann MD, Svendsen JH. Inflammation in the genesis and perpetuation of atrial fibrillation. Eur Heart $\mathrm{J} 2005$; 26: 2083-92. https://doi.org/10.1093/eurheartj/ehi350

Furie KL. High-dose statins should only be used in atherosclerotic strokes. Stroke 2012; 43: 1994-5. https://doi.org/10.1161/STROKEAHA.111.633339

Gagné C, Bays HE, Weiss SR, Mata P, Quinto K, Melino M, et al. Efficacy and safety of ezetimibe added to ongoing statin therapy for treatment of patients with primary hypercholesterolemia. Am J Cardiol 2002; 90: 1084-91. https://doi.org/10.1016/S0002-9149(02)02774-1

Ghebleh Zadeh N, Vaezi G, Bakhtiarian A, Mousavi Z, Shiravi A, Nikoui V. The potassium channel blocker, dalfampridine diminishes ouabain-induced arrhythmia in isolated rat atria. Arch Physiol Biochem 2019; 125: 25-
29. https://doi.org/10.1080/13813455.2018.1430158

Guo J, Zhang WZ, Zhao Q, Wo JS, Cai SL. Study on the effect of different doses of rosuvastatin on ventricular remodeling in patients with acute coronary syndrome after emergency percutaneous coronary intervention. Eur Rev Med Pharmacol Sci 2017; 21: 4457-4463.

$\mathrm{Hu}$ X, Cheng J, Li C. Effects of rosuvastatin and atorvastatin on nonsustained ventricular tachycardia in patients with ST-elevation myocardial infarction: a retrospective analysis. Eur J Clin Pharmacol 2018; 74: 29-35. https://doi.org/10.1007/s00228-017-2338-8

Ishida $K$, Kimura $F$, Imamaki $M$, Ishida $A$, Shimura $H$, Kohno $\mathrm{H}$, et al. Relation of inflammatory cytokines to atrial fibrillation after off-pump coronary artery bypass grafting. Eur J Cardiothorac Surg 2006; 29: 501-5. https://doi.org/10.1016/j.ejcts.2005.12.028

Jain MK, Ridker PM. Anti-inflammatory effects of statins: clinical evidence and basic mechanisms. Nat Rev Drug Discov 2005; 4: 977-87. https://doi.org/10.1038/nrd1901

Jones $\mathrm{PH}$, Davidson MH, Stein EA, Bays HE, McKenney $\mathrm{JM}$, Miller $\mathrm{E}$, et al. Comparison of the efficacy and safety of rosuvastatin versus atorvastatin, simvastatin, and pravastatin across doses (STELLAR* Trial). Am J Cardiol 2003; 92: 152-60. https://doi.org/10.1016/ S0002-9149(03)00530-7

Kayikcioglu M, Can L, Evrengul H, Payzin S, Kultursay $\mathrm{H}$. The effect of statin therapy on ventricular late potentials in acute myocardial infarction. Int $\mathrm{J}$ Cardiol 2003; 90: 63-72. https://doi.org/10.1016/S0167-5273(02)00516-8

Kowalewski M, Urban M, Mroczko B, Szmitkowski M. Proinflammatory cytokines (IL-6, TNF-alpha) and cardiac troponin I (cTnl) in serum of young people with ventricular arrhythmias. Pol Arch Med Wewn 2002; 108: 647-51.

Law MR, Wald NJ, Rudnicka AR. Quantifying effect of statins on low density lipoprotein cholesterol, ischaemic heart disease, and stroke: systematic review and metaanalysis. BMJ 2003; 326: 1423. https://doi.org/10.1136/ bmj.326.7404.1423

Lenderink T, Boersma E, Gitt AK, Zeymer U, Wallentin L, Van de Werf $F$, et al. Patients using statin treatment within $24 \mathrm{~h}$ after admission for ST-elevation acute coronary syndromes had lower mortality than nonusers: a report from the first Euro Heart Survey on acute coronary syndromes. Eur Heart J 2006; 27: 1799804. https://doi.org/10.1093/eurheartj/ehl125

Libby $\mathrm{P}$, Aikawa M. Mechanisms of plaque stabilization with statins. Am J Cardiol 2003; 91: 4-8. https://doi.org/10.1016/S0002-9149(02)03267-8

Lima JA, Desai MY, Steen H, Warren WP, Gautam S, Lai S. Statin-induced cholesterol lowering and plaque regression after 6 months of magnetic resonance imaging-monitored therapy. Circulation 2004; 110: 2336-41. https://doi.org/10.1161/01.CIR.0000145170. 22652.51

London B, Baker LC, Lee JS, Shusterman V, Choi BR, Kubota $T$, et al. Calcium-dependent arrhythmias in transgenic mice with heart failure. Am J Physiol Heart C 2003; 284: H431-41. https://doi.org/10.1152/ajpheart. 
00431.2002

Marcus GM, Smith LM, Ordovas K, Scheinman MM, Kim AM, Badhwar $\mathrm{N}$, et al. Intracardiac and extracardiac markers of inflammation during atrial fibrillation. Heart Rhythm 2010; 7: 149-54. https://doi.org/10.1016/j.hrthm. 2009.10.004

Matsumori A, Ono K, Nishio R, Nose Y, Sasayama S. Amlodipine inhibits the production of cytokines induced by ouabain. Cytokine 2000; 12: 294-7. https://doi.org/ 10.1006/cyto.1999.0555

Mehrzadi S, Kamrava SK, Dormanesh B, Motevalian M, Hosseinzadeh A, Hosseini Tabatabaei SM, et al. Melatonin synergistically enhances protective effect of atorvastatin against gentamicin-induced nephrotoxicity in rat kidney. Can J Physiol Pharm 2016; 94: 265-71. https://doi.org/10.1139/cjpp-2015-0277

Moradi S, Nikoui V, Imran Khan M, Amiri S, Jazaeri F, Bakhtiarian A. Involvement of inflammatory cytokines in antiarrhythmic effects of clofibrate in ouabain-induced arrhythmia in isolated rat atria. Adv pharmacol sci 2016; 2016: 9128018. https://doi.org/10.1155/2016/9128018

Najjari M, Vaezi G, Hojati V, Mousavi Z, Bakhtiarian A, Nikoui V. Involvement of IL-1 $\beta$ and IL-6 in antiarrhythmic properties of atorvastatin in ouabaininduced arrhythmia in rats. Immunopharm Immunot 2018; 40: 256-61. https://doi.org/10.1080/08923973. 2018.1440592

Nunes JP. Statins in primary prevention: impact on mortality. A meta-analysis study. Minerva Cardioangiol 2017; 65: 531-8.

O'Gara PT, Kushner FG, Ascheim DD, Casey DE, Chung MK, De Lemos JA, et al. 2013 ACCF/AHA guideline for the management of ST-elevation myocardial infarction: executive summary: a report of the American College of Cardiology Foundation/American Heart Association Task Force on Practice Guidelines. J Am Coll Cardiol 2013; 61: 485-510. https://doi.org/10.1016/j.jacc.2012. 11.018

Pehlivanidis AN, Athyros VG, Demitriadis DS, Papageorgiou AA, Bouloukos VJ, Kontopoulos AG. Heart rate variability after long-term treatment with atorvastatin in hypercholesterolaemic patients with or without coronary artery disease. Atherosclerosis 2001; 157: 463-9. https://doi.org/10.1016/S0021-9150(00) 00746-2

Psychari SN, Apostolou TS, Sinos L, Hamodraka E, Liakos G, Kremastinos DT. Relation of elevated C-reactive protein and interleukin- 6 levels to left atrial size and duration of episodes in patients with atrial fibrillation. Am J Cardiol 2005; 95: 764-7. https://doi.org/10.1016/ j.amjcard.2004.11.032

Rodrigues-Mascarenhas S, Dos Santos NF, Rumjanek VM. Synergistic effect between ouabain and glucocorticoids for the induction of thymic atrophy. Biosci Rep 2006; 26 : 159-69. https://doi.org/10.1007/s10540-006-9012-1

S Antonopoulos A, Margaritis M, Lee R, Channon K, Antoniades C. Statins as anti-inflammatory agents in atherogenesis: molecular mechanisms and lessons from the recent clinical trials. Curr Pharm Des 2012; 18:
1519-1530. https://doi.org/10.2174/1381612127995048 03

Sanchez-Quinones J, Marin F, Roldan V, Lip GY. The impact of statin use on atrial fibrillation. QJM Int J Med 2008; 101: 845-61. https://doi.org/10.1093/qjmed/ hen 101

Sever PS, Dahlöf B, Poulter NR, Wedel H, Beevers G, Caulfield $\mathrm{M}$, et al. Prevention of coronary and stroke events with atorvastatin in hypertensive patients who have average or lower-than-average cholesterol concentrations, in the Anglo-Scandinavian Cardiac Outcomes Trial-Lipid Lowering Arm (ASCOT-LLA): a multicentre randomised controlled trial. The Lancet 2003; 361: 1149-58. https://doi.org/10.1016/S01406736 (03) $12948-0$

Sicard P, Zeller M, Dentan G, Laurent Y, Touzery C, L'huillier I, Janin-Manificat $L$, et al. Beneficial effects of statin therapy on survival in hypertensive patients with acute myocardial infarction: data from the RICO survey. Am J Hypertens 2007; 20: 1133-9.

Tokazzabani Belasi F, Vaezi G, Bakhtiarian A, Hojati V, Mousavi Z, Nikoui V. On the benefit of melatonin in protection against ouabain-induced arrhythmia through modulation of oxidative stress factors in isolated rat atria. Biol Rhythm Res 2018; 49: 772-81. https://doi.org/ 10.1080/09291016.2018.1424769

Turagam MK, Downey FX, Kress DC, Sra J, Tajik AJ, Jahangir A. Pharmacological strategies for prevention of postoperative atrial fibrillation. Expert Rev Clin Pharmacol 2015; 8: 233-250. https://doi.org/10.1586/ 17512433.2015.1018182

Upala S, Wirunsawanya K, Jaruvongvanich V, Sanguankeo A. Effects of statin therapy on arterial stiffness: a systematic review and meta-analysis of randomized controlled trial. Int J Cardiol 2017; 227: 338-41. https://doi.org/10.1016/j.ijcard.2016.11.073

Vasan RS, Sullivan LM, Roubenoff R, Dinarello CA, Harris T, Benjamin EJ, et al. Inflammatory markers and risk of heart failure in elderly subjects without prior myocardial infarction: the Framingham Heart Study. Circulation 2003; 107: 1486-91. https://doi.org/10.1161/01.CIR. 0000057810.48709.F6

Vila N, Castillo J, Dávalos A, Chamorro A. Proinflammatory cytokines and early neurological worsening in ischemic stroke. Stroke 2000; 31: 2325-9. https://doi.org/10.1161/ 01.STR.31.10.2325

Vrtovec B, Okrajsek R, Golicnik A, Ferjan M, Starc V, Radovancevic B. Atorvastatin therapy increases heart rate variability, decreases QT variability, and shortens QTc interval duration in patients with advanced chronic heart failure. J Card Fail 2005; 11: 684-90. https://doi. org/10.1016/j.cardfail.2005.06.439

Wallace JL, McKnight W, Reuter BK, Vergnolle N. NSAIDinduced gastric damage in rats: requirement for inhibition of both cyclooxygenase 1 and 2 . Gastroenterology 2000; 119: 706-14. https://doi.org/ 10.1053/gast.2000.16510

Watanabe H, Tanabe N, Watanabe T, Darbar D, Roden DM, Sasaki S, et al. Metabolic syndrome and risk of 
development of atrial fibrillation: the Niigata preventive medicine study. Circulation 2008; 117: 1255-1260. https://doi.org/10.1161/CIRCULATIONAHA.107.744466

Weitz-Schmidt G. Statins as anti-inflammatory agents. Trends Pharmacol Sci 2002; 23: 482-7. https://doi.org/ 10.1016/S0165-6147(02)02077-1

Wilson PW, D'Agostino RB, Levy D, Belanger AM, Silbershatz $\mathrm{H}$, Kannel WB. Prediction of coronary heart disease using risk factor categories. Circulation 1998; 97: 1837-47. https://doi.org/10.1161/01.CIR.97.18.1837
Wu N, Xu B, Xiang Y, Wu L, Zhang Y, Ma X, et al. Association of inflammatory factors with occurrence and recurrence of atrial fibrillation: a meta-analysis. Int $J$ Cardiol 2013; 169: 62-72. https://doi.org/10.1016/ j.ijcard.2013.08.078

Yu SP, Choi DW. Na+-Ca2+ exchange currents in cortical neurons: concomitant forward and reverse operation and effect of glutamate. Eur J Neurosci 1997; 9: 127381. https://doi.org/10.1111/j.1460-9568.1997.tb01482.x 